\title{
Visualizing the perisaccadic shift of spatiotopic coordinates
}

\author{
WAYNE A. HERSHBERGER \\ Northerm Illinois University, De Kalb, Illinois \\ J. SCOTT JORDAN \\ Saint Xavier University, Chicago, Illinois \\ and \\ DONALD R. LUCAS \\ Northern Illinois University, De Kalb, Illinois
}

\begin{abstract}
A point light source flickering on and off during a horizontal saccade projects a horizontal array onto the retina. The apparent visual direction of the tail end of the perceived (phantom) array reflects the amount of perisaccadic shift of spatiotopic coordinates that has been completed by the end of the saccade. Four men, saccading $8^{\circ}$ to the right across a flashing light, judged the horizontal visual direction of the left (tail) end of the phantom array relative to the left end of a standard $8^{\circ}$ array that had projected an image onto the retina before the saccade began. On average, the left ends appeared to be aligned when the last flash in the phantom array was imaged on the retina $7.4^{\circ}$ to the right of the image of the left end of the standard array. This result implies that the shift of spatiotopic coordinates is virtually complete by the end of the saccade.
\end{abstract}

A point light source flickering on and off during a saccade projects a pattern onto the retina. The perceived pattern differs from the retinal pattern, with this difference reflecting the perisaccadic shift of spatiotopic coordinates. Hershberger (1987) characterized the perceptual pattern as a phantom array, fixed in space, with the sequential order of the flashes creating an impression of apparent motion within the array. Figure 1 illustrates the phantom array, as Hershberger first described it. Note that the phantom array is only about half as long as the retinal array (or, saccade) and is displaced in the direction of the saccade.

Hershberger and Jordan (in press) found that theoretically naive observers see it this way as well: They tested 75 undergraduates who responded to 12 forced-choice questions regarding flashes they saw while saccading horizontally across a light-emitting diode (LED) flashing in the dark at a frequency of $200 \mathrm{~Hz}$ (a 1 -msec flash every $5 \mathrm{msec}$ ); each flash had a luminance of about $50 \mathrm{~cd} / \mathrm{m}^{2}$. The questions were designed to determine in what direction the flashes appeared to move and/or be displaced. All but 2 of the 75 naive observers consistently saw a horizontal row of flashing dots whenever they saccaded horizontally across the flashing LED. Furthermore,

\footnotetext{
A preliminary report of this research was presented at the Annual Meeting of the Association for Research in Vision and Ophthalmology, Ft. Lauderdale, FL, May 1995. The authors thank Associate Editor Robert P. O'Shea and three anonymous referees for helpful comments on an earlier version of the manuscript. Correspondence should be addressed to W. A. Hershberger, Department of Psychology, Northern Illinois University, De Kalb, IL 60115 (e-mail: wah@ @iu.edu).
}

all but 5 of these 73 observers saw the individual dots as being stationary or as moving in a direction opposite to the eye movement's (this includes the phi produced by the sequential order of the flashes). Also, 63 of these 73 observers consistently saw the first flash in the array as being displaced toward the new direction of gaze. Finally, 57 of these 63 observers reported that the entire array appeared to be located to that side of the LED associated with the new direction of gaze. Irrespective of whether the minority of incongruent answers represent fundamental individual differences or merely experimental noise, it is clear that theoretical naivete does not preclude an observer's experiencing the phantom array as Hershberger described it.

The most remarkable feature of the phantom array is the discrete displacement of the array in the direction of the eye movement, with the first flash being displaced the most. Despite the fact that the angular displacement of the first flash is about half the size of the attendant saccade, this displacement develops fully during a single interflash interval. The size of the first flash's displacement, relative to the size of the saccade, reflects the proportion of the overall shift of spatiotopic coordinates that has occurred before the saccade begins. Because the LED in Hershberger and Jordan's (in press) experiment flashed once every $5 \mathrm{msec}$, it is apparent that the discrete portion of the overall shift is very rapid, having a time constant that is less than $5 \mathrm{msec}$. This discrete half of the overall spatiotopic shift also occurs very early. Jordan and Hershberger (1994) found that the first-displaced flash in the phantom array is one that falls upon the retina at least 80 msec before the eye actually begins to rotate. 


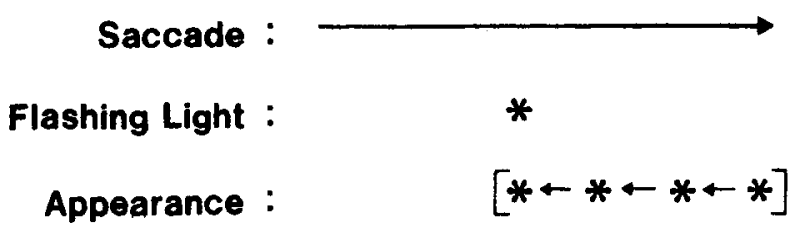

Figure 1. If you shift your gaze saccadically from the left to the right of a point light source in a darkened room, blinking on and off at $120 \mathrm{~Hz}$, you will see phi movement to the left within a phantom array that is displaced to the right. From "Saccadic Eye Movements and the Perception of Visual Direction," by W. [A.] Hershberger, 1987, Perception \& Psychophysics, 4I, p. 39. Copyright 1987 by Psychonomic Society, Inc. Reprinted with permission.

The fact that the left end of the array, as illustrated in Figure 1, appears roughly coincident, spatially, with the flashing LED implies that the overall spatiotopic shift is virtually complete by the time that the optical transient (eye movement) has registered fully. However, in order to quantify the percentage of the shift actually completed, it is necessary to determine the apparent location of the left end of the array more precisely. This was done in the present experiment by adjusting the horizontal position of an LED (that flashed at $500 \mathrm{~Hz}$ during a rightward saccade) so that the left end of the resulting phantom array appeared to be vertically aligned with the left end of a static, standard array that had projected an image upon the retina at a known location well before the saccadic eye movement.

\section{METHOD}

\section{Participants}

Four men (D.L., S.C., S.J., and W.H.) participated as psychophysical observers. Each observer participated in 200 trials. D.L. and S.C. wore contact lenses to correct for myopia; W.H. wore contact lenses to correct for presbyopia. S.C. was naive with respect to the theoretical hypothesis.

\section{Apparatus}

Sixteen LEDs were mounted on a display panel in three horizontal rows as depicted in Figure 2 . The top and bottom rows were $1^{\circ}$ from the center row. The two LEDs marked $F$ (fixation) and $T$ (saccadic target) were red, the others green. The luminance of $F$ and $T$ were $30 \mathrm{~mL}$, and the luminance of the LEDs in the top and bottom row were 6 and $60 \mathrm{~mL}$, respectively; the LEDs in the top row were set dimmer because they all flashed at once. The display panel was "centered" $150 \mathrm{~cm}$ in front of the participant's monitored right eye, with $\mathrm{F}$ and $\mathrm{T} 4^{\circ}$ to the left and right of the eye's primary position (i.e., straight ahead). At this distance, each LED subtended $0.2^{\circ}$. An infrared eye-movement monitor (Applied Science Laboratories EyeTrac 200) was used to track the horizontal movements of the participant's right eye. The Eye-Trac is accurate to within $0.25^{\circ}$, and it has a response time of $4 \mathrm{msec}$. The Eye-Trac was calibrated before every trial as the participant fixated $F$ and $T$ in turn. A 486 IBM computer with a 12-bit IBM a/d converter energized target LEDs and digitized the output of the eye-movement monitor at $1000 \mathrm{~Hz}$.
\end{abstract}

\section{Procedure}

On each trial, $T$ came on for $10 \mathrm{msec}, 50 \mathrm{msec}$ after $F$ went off, whereupon the participant saccaded in the dark from $F$ to $T$. Two ar- rays were projected onto the participant's retina on each trial, each array subtending about $8^{\circ}$. One (the static retinal array) was projected during the saccadic latency when all nine Os flashed on for $1 \mathrm{msec}, 50 \mathrm{msec}$ after $\mathrm{T}$ went off (i.e., long before the eye began to move). The other array (the dynamic retinal array) was projected onto the retina during the saccade as one (and only one) of the five $\mathrm{Xs}$ blinked on and off at $500 \mathrm{~Hz}$ for the duration of the saccade. The end of the saccade was determined very precisely. The computer sampled the Eye-Trac signal once each millisecond and compared that value with the previous one; when the difference dropped to or below zero, the blinking $\mathrm{X}$ was disabled. The particular $\mathrm{X}$ used on a given trial was varied randomly, with the restriction that each $X$ was used four times in each block of 20 trials.

Following each saccade, the participant (now in total darkness) indicated whether the left end of the phantom array (the comparison stimulus) had appeared to be to the left of, to the right of, or aligned with the left end of the array of Os (the standard stimulus). Using these data, a point of subjective equality (PSE) was calculated for each participant according to the standard method of constant stimuli. The PSE, in the present case, is the physical location that an $\mathrm{X}$ would have to occupy (measured here in terms of its horizontal distance from the left end of the array of Os) in order for the left end of the phantom array projected by that $X$ to be judged subjectively equal to (aligned with) the left end of the array of Os. With the left end of the array of Os located at 0, the PSE is also the constant error (CE = PSE - Standard). The amount of spatiotopic shift that has occurred by the end of the saccade can be calculated by subtracting the $\mathrm{CE}$ from the size of the average saccade.

\section{RESULTS}

The results are summarized in Table 1 and illustrated in Figure 3. For the average participant, there was a $7.4^{\circ}$ shift of the spatiotopic coordinates during the average $7.9^{\circ}$ saccade, so approximately $94 \%$ of the overall shift was completed by the end of the saccade. This means that, with the left end of the distal static array projecting onto the retina $4^{\circ}$ to the left of the fovea, the flash at the left end of the distal phantom array (i.e., the last flash in the array) had to project onto a retinal locus $3.4^{\circ}$ to the right of the fovea in order for the two distal visual arrays to appear to have their left ends aligned. Because the spatial polarity of distal and proximal visual arrays are reversed, the left ends of the distal phenomenal arrays correspond to the right ends of the proximal retinal arrays. Figure 3 shows where the proximal dynamic array (the source of the phantom array) had to be located on the retina in order for its right end to share the same spatiotopic coordinates as the right end of the static retinal array. Figure 3 also shows that there was a $7.4^{\circ}$ shift of the spatiotopic coordinates during the average $7.9^{\circ}$ saccade. Furthermore, if Deubel and Bridgeman's (1995) suggested correction for the perceptual effects of lens overshoot at the end of an $8^{\circ}$ saccade is taken into account $(3 \%)$, the percentage of the overall shift that was complete by the end of the saccade appears to be closer to $97 \%$.

\section{DISCUSSION}

By using the phantom array to visualize the perisaccadic shift of spatiotopic coordinates, we have found com- 


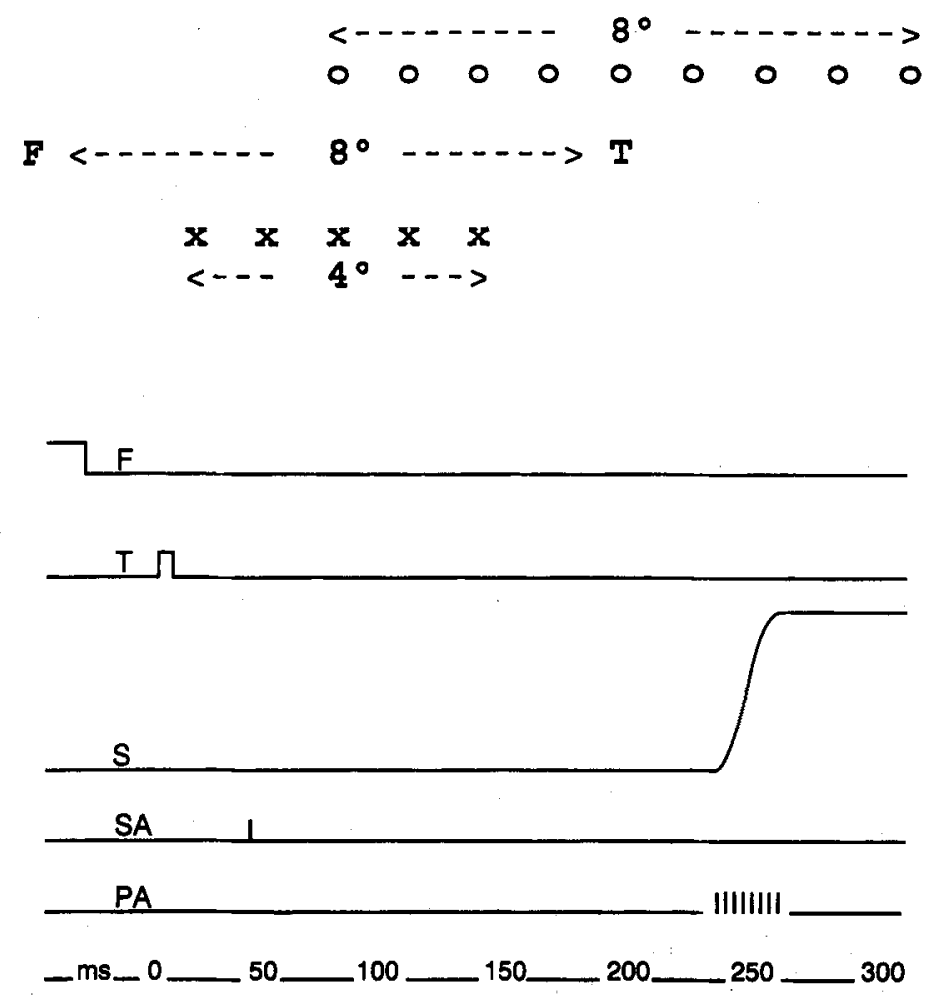

Figure 2. Top panel: Arrangement of the 16 LEDs on the display panel. $F$ and $T$ represent the fixation and saccadic targets, respectively. The nine Os represent the LEDs comprising the static array that flashed all at once during the saccadic latency. The five $X s$ represent the five possible positions of the blinking LED that generated the dynamic phantom array during the saccade. Bottom panel: Chronology of each trial. $F$ is the fixation target. $T$ is the saccadic target. $S$ is the saccade from $F$ to $T$. SA is the standard array (all nine Os). PA is the phantom array (one of the five $\mathrm{Xs}$ ). The bottom trace represents elapsed time in milliseconds, relative to $T$ onset.

pelling evidence that the overall spatiotopic shift is virtually complete $(94 \%-97 \%)$ by the end of the saccade. That is, the shift of spatiotopic coordinates occasioned by a saccade is effectively complete by the time that an image projected onto the retina at the end of the saccade is perceptually registered. This undoubtedly helps contribute to perceptual visual-direction constancy. It does not, however, imply that a flash from a stationary light source after a saccade will necessarily appear to share the same absolute (egocentric) visual direction as a presaccadic flash from that same stationary source. The participants in the present experiment were comparing the relative visual direction of two retinal arrays, and they were not required to judge the absolute visual direction of either array. Had they been estimating the absolute visual direction of the last flash in the phantom array relative to the absolute visual direction of a remembered presaccadic flash, the present findings would likely resemble

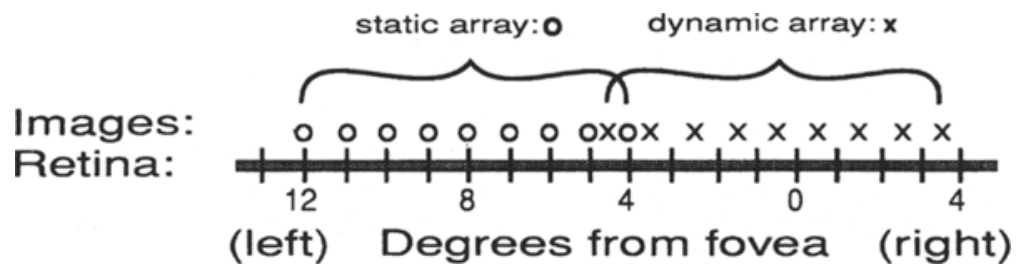

Figure 3. Location of the two retinal arrays at the point of subjective equality. The right end of the $X$ array marks the retinal location where a flash at the end of a $7.9^{\circ}$ saccade must be imaged in order for it to appear to lie in the same visual direction as a presaccadic flash imaged on the retina at the location marked by the right end of the $O$ array. 
those of prior experiments in which participants mislocalized point sources flashed long (e.g., $100 \mathrm{msec}$ ) after a saccade had terminated (e.g., see Matin, 1972). Skavenski (1976) has observed that these belated errors are probably attributable to a degraded memory image of the extraretinal signal involved in fixating a presaccadic fixation stimulus that serves as a reference point for the belated judgments (also see Hershberger, 1987). However, all that is beyond the scope of the present study. So too are the saccade-contingent mislocalizations that occur when targets are flashed upon an illuminated background (e.g., Bischof \& Kramer, 1968; Honda, 1993; Mateeff, 1978; O'Regan, 1984), because such mislocalizations occur even when the background is moved, rather than the eye (MacKay, 1970).

The focus of the present study was the timing of the perisaccadic shift of spatiotopic coordinates. The present participants were asked to judge only the relative visual direction (i.e., the apparent alignment or misalignment) of two retinal arrays - one projected during the saccade, and another projected $130-240 \mathrm{msec}$ before the saccade began, depending on the participant's saccadic latency (see Table 1). This was early enough to predate the perisaccadic shift of spatiotopic coordinates, which commences about $80 \mathrm{msec}$ before the saccade begins (Jordan \& Hershberger, 1994), but late enough to allow the participants to judge the spatial alignment of the two retinal arrays.

The perisaccadic shift of spatiotopic coordinates that begins early ( $80 \mathrm{msec}$ before the saccade) is responsible for the abrupt displacement of the first flash in the phantom array. That is, the presaccadic component of the overall shift of spatiotopic coordinates - the part that is responsible for the discrete displacement of the first flash in the phantom array-is very fast, having a time constant that is less than $5 \mathrm{msec}$. We know that the time constant is less than $5 \mathrm{msec}$ because, as noted above, Hershberger and Jordan (in press) used a point light source that was continuously present and flashing at $200 \mathrm{~Hz}$ (i.e., once every $5 \mathrm{msec}$ - - thus, the spatiotopic shift that displaced the first flash took less than $5 \mathrm{msec}$.

In addition to being very fast, the abrupt displacement of the first flash is also very large, being roughly half the size of the attendant saccade. Both the size and the speed, as well as the timing, of the early discrete com- ponent of the spatiotopic shift can be seen in Figure 4, taken from Jordan and Hershberger (1994). In that experiment, as in the present one, an LED flashing at $500 \mathrm{~Hz}$ generated a phantom array whenever a participant observer $(n=4)$ saccaded $\left(8^{\circ}\right.$, from left to right) across it. This blinking LED was vertically bracketed by a pair of LEDs that flashed once each trial (for $1 \mathrm{msec}$ ) at a predetermined time before, during, or after the saccade. The $1-\mathrm{msec}$ flash was a marker. The participant's task was to judge the spatial position of the marker on a 5 -point scale, with 5 representing the right (front) end of the phantom array and 1 representing the left (tail) end of the array. Figure 4 shows the change in the observers' average marker-position judgments (MPJs) as a function of the time of the marker flash, relative to the onset of the saccade (marker-saccade asynchrony). Figure 4 also shows the time course of the entire perisaccadic shift of spatiotopic components; it begins abruptly $80 \mathrm{msec}$ before the saccade, and it is virtually complete by the end of the $8^{\circ}$ saccade $120 \mathrm{msec}$ later.

The received view, held for the better part of 30 years (see Matin, 1972), is that the perisaccadic shift of the retina's spatiotopic coordinates is sluggish, forming, as it were, one long continuous change that temporally brackets the attendant saccade. Although the overall shift does indeed appear to be prolonged, the phantom array indicates that this overall shift is discontinuous, comprising two components (at least), the first of which is extremely abrupt. The second putative component mediates the remainder of the shift, virtually completing the process by the end of the saccade.

It is our thesis that the two parts of the overall shift (i.e., the first half and the second half) are mediated by two different eye-position signals, both efferent. We are not the first to consider a hybrid eye-position signal. However, all others have involved afference-efference hybrids (Matin, 1972, 1976a, 1976b; Shebilske, 1977; Skavenski, 1972, 1976; Steinbach, 1987). Invoking Robinson's (1975) closed-loop model of the oculomotor system, we suggest that the first eye-position signal is the oculomotor system's reference signal specifying intended eye orientation, and that the second is an efference copy representing actual eye orientation. We are using the expression "efference copy" as Robinson did in describing his closed-loop model of the oculomotor sys-

Table 1

PSE (CE) and Shift of Spatiotopic Coordinates for Average Saccades

\begin{tabular}{|c|c|c|c|c|c|c|c|c|}
\hline \multirow[b]{3}{*}{ Participant } & \multirow[b]{3}{*}{ PSE (CE) } & \multirow[b]{3}{*}{ Shift } & \multicolumn{6}{|c|}{ Saccade } \\
\hline & & & \multicolumn{2}{|c|}{ Size } & \multicolumn{2}{|c|}{ Latency } & \multicolumn{2}{|c|}{ Duration } \\
\hline & & & Average & $S D$ & Average & $S D$ & Average & $S D$ \\
\hline D.L. & $0.6^{\circ}$ & $7.5^{\circ}$ & $8.1^{\circ}$ & $1.0^{\circ}$ & 181 & 22 & 36 & 2 \\
\hline S.C. & $0.4^{\circ}$ & $7.2^{\circ}$ & $7.6^{\circ}$ & $1.0^{\circ}$ & 291 & 85 & 39 & 4 \\
\hline S.J. & $0.5^{\circ}$ & $7.6^{\circ}$ & $8.1^{\circ}$ & $1.8^{\circ}$ & 216 & 38 & 47 & 8 \\
\hline W.H. & $0.4^{\prime \prime}$ & $7.4^{\circ}$ & $7.8^{\circ}$ & $1.8^{\circ}$ & 234 & 29 & 34 & 4 \\
\hline Mean & $0.5^{\circ}$ & $7.4^{\prime \prime}$ & $7.9^{\circ}$ & & 231 & & 39 & \\
\hline$S D$ & $0.1^{\circ}$ & $0.2^{\prime \prime}$ & $0.3^{\circ}$ & & 46 & & 6 & \\
\hline
\end{tabular}

Note-PSE, point of subjective equality; CE, constant error. Latency and duration are in milliseconds. 


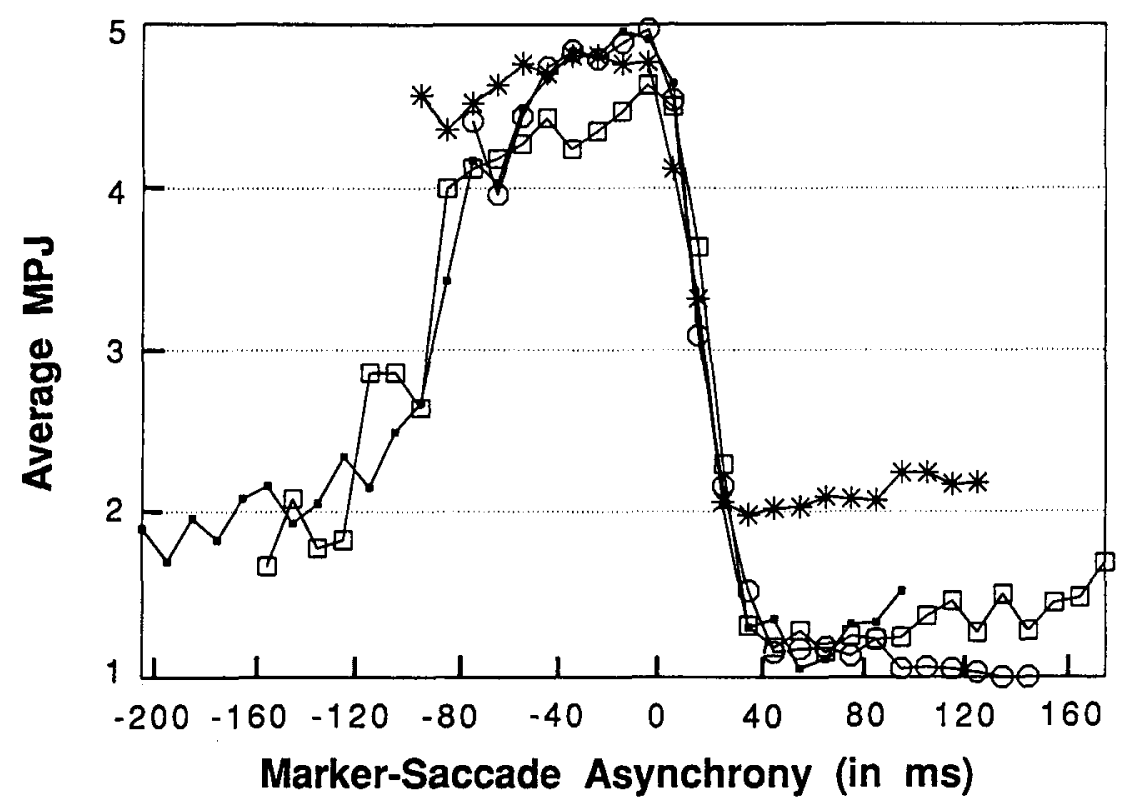

- - Observer $1 \rightarrow$ Observer $2 *$ Observer 3 -O-Observer 4

Figure 4. The observers' average marker-position judgments (MPJs) plotted as a function of the marker-saccade asynchrony. From "Timing the Shift in Retinal Local Signs That Accompanies a Saccadic Eye Movement," by J. S. Jordan and W. A. Hershberger, 1994, Perception \& Psychophysics, 55, p. 661. Copyright 1994 by Psychonomic Society, Inc. Reprinted with permission.

tem. Robinson's closed-loop model of the oculomotor system utilizes two separate indices of the variable being controlled, as do all servo systems: a feedback signal and a reference signal. The feedback signal in Robinson's model is an efference copy; it comprises corollary discharges from premotorneurons in the brainstem indicating actual eye position. The reference signal in Robinson's model is a centralized command signal that specifies the intended value of the feedback signal-that is, intended eye position. Regrettably, these two types of neural signals (commands and copies) are frequently confused in the literature, perhaps because von Holst and Mittelstaedt (1950) did not fully appreciate the importance of the difference when they first coined the expression $e f$ ference copy, as Mittelstaedt (1958) later acknowledged. However, there is a real difference between them, a difference that is reflected in the perisaccadic illusion of visual direction.

To summarize, our thesis is this: We adopt the received view that the spatiotopic coordinates for registering retinal input are determined by an extraretinal eyeposition signal. Furthermore, we adopt the received view that the extraretinal eye-position signal is oculomotor. We also adopt the received view that the oculomotor system is a closed-loop control system incorporating two oculomotor signals, both of which correspond to eye orientation (reference signal and efference copy). Thus, we are faced with the problem of identifying which of the two oculomotor candidates actually serves as the extraretinal signal. Our proposed solution to this conundrum is the parsimonious suggestion that the extraretinal signal is simply an average of the two oculomotor signals. For perisaccadic shifts of spatiotopic coordinates, our thesis implies that (1) changes in each type of signal should contribute about one half of the overall perisaccadic shift, (2) the first half should be discrete and occur before the eyes move, and (3) the last half should be continuous and occur during the actual eye movement. The phenomenon known as the phantom array fits all three of these hypotheses exactly.

Because the perisaccadic shift of spatiotopic coordinates is $94 \%-97 \%$ complete by the time that lights flashed at the end of a saccade are perceptually registered, and because retinal processing of visual input takes time (at least $40 \mathrm{msec}$ ), it appears that the second component of the extraretinal signal would have to be a slightly damped version of the efference copy, which is itself time locked to the eye movement.

Our present findings (involving perceptual judgments) are generally consistent with the well-documented finding that saccades directed toward perisaccadic targets flashed in the dark are dysmetric (Dassonville, Schlag, \& Schlag-Rey, 1992, 1995; Hershberger \& Lucas, 1993, 1994; Honda, 1990; Schlag \& Schlag-Rey, 1995; however, cf. Hallett \& Lightstone, 1976a, 1976b). The parallel is not perfect, however, because the oculomotor findings are 
not entirely consistent, even excepting Hallett and Lightstone's data. Although oculomotor errors of localization for intrasaccadic targets (ITs) flashed at the beginning of an ongoing saccade $\left(\mathrm{S}_{0}\right)$ are always found to be positive (i.e., in the direction of $\mathrm{S}_{0}$ ) and declining in magnitude, the magnitude and polarity of the errors for targets flashed at the end of $S_{0}$ tend to vary from individual to individual. For instance, one of Honda's three participants (K.S.) had small positive errors, whereas another (H.H.) had large negative errors for targets flashed at the end of $S_{0}$ (see the $S_{1}$ data-saccades to the ITs-in Figure 20.4 of Honda, 1990). Furthermore, when two of the present participants (W.H. and S.C.) were tested using Honda's oculomotor paradigm (Hershberger \& Lucas, 1994), their data also differed; W.H.'s data were like K.S.'s, and S.C.'s were like H.H.'s. However, in the present psychophysical experiment, W.H.'s and S.C.'s data both indicate that the shift of spatiotopic coordinates was at least $94 \%$ complete by the end of $S_{0}$. Apparently, S.C.'s relatively large negative oculomotor errors for ITs flashed at the end of $S_{0}$ (see Hershberger \& Lucas, 1994) reflect an idiosyncratic response bias that does not depend on the perceptual judgments of target location tapped by the present psychophysical method. Honda (1990) reported finding a close correspondence between perceptual and oculomotor localization errors; however, he was talking not about single saccades $\left(S_{1}, s\right)$ to ITs flashed during $S_{0}$ but, rather, about the direction of gaze after the eye had moved twice-that is, after it had "caught" the perceptually displaced target with a third saccade $\left(\mathrm{S}_{2}\right)$. K.S.'s perceptual errors corresponded to his oculomotor localization errors at the end of $S_{2}$, not at the end of $S_{1}$.

Although perisaccadic targets flashed at the end of $S_{0}$ yield perceptual and oculomotor effects that may differ, perisaccadic targets flashed before $S_{0}$ yield perceptual and oculomotor effects that may be similar - a similarity that is readily obscured by statistical manipulations of the data. For instance, when Schlag and Schlag-Rey (1995) flashed a single saccadic target (located $17^{\circ}$ to the right of fixation) twice in succession before the eye could move, the latter flash sometimes appeared to be displaced to the right of the first flash, and the saccade to the first flash was sometimes followed by a second saccade also to the right. When Schlag and Schlag-Rey plotted the amplitude of each of these second saccades as a function of the time of the eliciting second flash, the results were, and are, revealing. It appears, from visual inspection of the four scatterplots in their Figure 6 (Schlag \& Schlag-Rey, 1995), that the data for each participant could be fit by a regression line having zero slope and a substantial $y$ intercept. In other words, the amplitude of the second saccade was substantial, and it did not vary as a function of time-reflecting a discrete step change. From visual inspection, the mean amplitude ranged from about $4^{\circ}$ (Figure $6 \mathrm{a}$ ) to about $8^{\circ}$ (Figure $6 \mathrm{c}$ ).

Although Schlag and Schlag-Rey (1995) plotted these important data, they did not interpret them. Instead, they combined them with data from trials on which no sec- ond saccade occurred and computed sliding averagesa procedure guaranteed to misrepresent step changes as ramps (see Hershberger, 1987). Eschewing this misleading mathematical algorithm, we submit, citing their own raw data as evidence, that the extraretinal-signal transient mediating Schlag and Schlag-Rey's "second saccades" was a step, not a ramp.

\section{REFERENCES}

BisChOF, N., \& KRAMER, E. (1968). Untersuchung und Überlegungen zur Richtungswahrnehmung bei willkürlichen sakkadischen Augenbewegungen [Research and theory on space perception and voluntary saccadic eye movements]. Psychologische Forschung, 32, 185218.

Dassonville, P., Schlag, J., \& Schlag-Rey, M. (1992). Oculomotor localization relies on a damped representation of saccadic eye displacement in human and nonhuman primates. Visual Neuroscience, 9, 261-269.

Dassonville, P., Schlag, J., \& Schlag-Rey, M. (1995). The use of egocentric and exocentric location cues in saccadic programming. $\mathrm{Vi}$ sion Research, 35, 2191-2199.

Devbel, H., \& Bridgeman, B. (1995). Perceptual consequences of ocular lens overshoot during saccadic eye movements. Vision Research, 35, 2897-2902.

Hallett, P. E., \& Lightstone, A. D. (1976a). Saccadic eye movements towards stimuli triggered by prior saccades. Vision Research, 16, 99-106.

Hallett, P. E., \& Lightstone, A. D. (1976b). Saccadic eye movements to flashed targets. Vision Research, 16, 107-114.

Hershberger, W. [A.] (1987). Saccadic eye movements and the perception of visual direction. Perception \& Psychophysics, 41, 35-44.

Hershberger, W. A., \& Jordan, J. S. (in press). The phantom array: A perisaccadic illusion of visual direction. Psychological Record.

Hershberger, W. A., \& Lucas, D. R. (1993). Saccades to targets flashed during a prior saccade. Investigative Ophthalmology \& Visual Science, 34, 1498.

Hershberger, W. A., \& LuCAs, D. R. (1994). Saccades to bright targets flashed during a prior saccade. Investigative Ophthalmology \& Visual Science, 35, 2033.

Honda, H. (1990). Eye movements to a visual stimulus flashed before, during, or after a saccade. In M. Jeannerod (Ed.), Attention and performance XIII: Motor representation and control (pp. 567-582). Hillsdale, NJ: Erlbaum.

HoNDA, H. (1993). Saccade-contingent displacement of the apparent position of visual stimuli flashed on a dimly illuminated structured background. Vision Research, 33, 709-716.

Jordan, J. S., \& Hershberger, W. A. (1994). Timing the shift in retinal local signs that accompanies a saccadic eye movement. Perception \& Psychophysics, 55, 657-666.

MACKAY, D. M. (1970). Mislocation of test flashes during saccadic image displacements. Naiure, 227, 731-733.

MateEfF, S. (1978). Saccadic eye movements and localization of visual stimuli. Perception \& Psychophysics, 24, 215-224.

MATIN, L. (1972). Eye movements and perceived visual direction. In D. Jameson \& L. Hurvich (Eds.), Handbook of sensory physiology (Vol. 7, pp. 331-380). Heidelberg: Springer-Verlag.

MATIN, L. (1976a). A possible hybrid mechanism for modification of visual direction associated with eye movements-The paralysed eye experiment reconsidered. Perception, 5, 233-239.

Matin, L. (1976b). Saccades and extraretinal signal for visual direction. In A. Monty \& J. W. Senders (Eds.), Eye movements and psychological processes (pp. 205-219). Hillsdale, NJ: Erlbaum.

MitTelstaedT, H. (1958). The analysis of behavior in term of control systems. In B. Schaffner (Ed.), Group processes: Transactions of the Fifth Conference (pp. 45-84). New York: Josiah Macy, Jr., Foundation.

O'REGAN, J. K. (1984). Retinal versus extraretinal influences in flash localization during saccadic eye movements in the presence of a visible background. Perception \& Psychophysics, 36, i-14. 
RoBinson, D. A. (1975). Oculomotor control signals. In G. Lennerstrand \& P. Bach-y-Rita (Eds.), Basic mechanisms of ocular motility and their clinical implications (pp. 337-374). New York: Pergamon.

SCHLAG, J., \& SCHLAG-ReY, M. (1995). Illusory localization of stimuli flashed in the dark before saccades. Vision Research, 35, 2347-2357.

SHEBILSKE, W. L. (1977). Visuomotor coordination in visual direction and position constancies. In W. Epstein (Ed.), Stability and constancy in visual perception (pp. 23-69). New York: Wiley.

SKAVENSKI, A. A. (1972). Inflow as a source of extraretinal eye position information. Vision Research, 12, 221-229.

SKaVENSKI, A. A. (1976). The nature and role of extraretinal eye-position information in visual localization. In A. Monty \& J. W. Senders (Eds.), Eye movements and psychological processes (pp. 277-287). Hillsdale, NJ: Erlbaum.

Steinbach, M. J. (1987). Proprioceptive knowledge of eye position. Vision Research, 27, 1737-1744.

von Holst, E., \& MitTelstaedT, H. (1950). Das Reafferenzprinzip [The reafference principle]. Naturwissenshaften, 37, 464-476.

(Manuscript received October 8, 1996; revision accepted for publication January 12, 1997.) 